\title{
Amyloid PET in Dementia Syndromes: A Chinese Multicenter Study
}

\author{
Zhihong Shi ${ }^{1}$, Li-ping Fu ${ }^{2,3}$, Nan Zhang ${ }^{4}$, Xiaobin Zhao ${ }^{5}$, Shuai $\mathrm{Liu}^{1}$, Chuantao Zuo ${ }^{6}$, $\mathrm{Li} \mathrm{Cai}^{7}$, Ying Wang ${ }^{7}$, \\ Shuo $\mathrm{Gao}^{7}$, Lin $\mathrm{Ai}^{* 5}$, Yi-Hui Guan*6, Baixuan $\mathrm{Xu}^{* 2}$, and Yong Ji*1,8,9 \\ ${ }^{I}$ Tianjin Key Laboratory of Cerebrovascular and Neurodegenerative Diseases, Department of Neurology, Tianjin Dementia Institute, \\ Tianjin Huanhu Hospital, Tianjin, China; ${ }^{2}$ Department of Nuclear Medicine, 1st Medical Center, Chinese PLA (People's Liberation \\ Army) General Hospital, Beijing, China; ${ }^{3}$ Department of Nuclear Medicine, China-Japan Friendship Hospital, Beijing, China; \\ ${ }^{4}$ Department of Neurology, General Hospital of Tianjin Medical University, Tianjin, China; ${ }^{5}$ Department of Nuclear Medicine, \\ Beijing Tiantan Hospital, Capital Medical University, Beijing, China; ${ }^{6}$ PET Center, Huashan Hospital, Fudan University, Shanghai, \\ China; ${ }^{7}$ Department of PET-CT Diagnostics, Tianjin Medical University General Hospital, Tianjin, China; ${ }^{8}$ China National Clinical \\ Research Center for Neurological Diseases, Beijing, China; and ${ }^{9}$ Department of Neurology, Beijing Tiantan Hospital, Capital \\ Medical University, Beijing, China
}

Cerebral $\beta$-amyloid deposits and regional glucose metabolism assessed by PET are used to distinguish between Alzheimer disease (AD) and other dementia syndromes. In the present multicenter study, we estimated the prevalence of $\beta$-amyloid deposits on PET imaging in a wide variety of dementia syndromes and mild cognitive impairment $(\mathrm{MCl})$ within a memory clinic population. Methods: Of the 1,193 consecutive patients with cognitive impairment $(\mathrm{Cl})$ who received $1{ }^{11} \mathrm{C}$-PIB PET or ${ }^{18} \mathrm{~F}-\mathrm{AV} 45$ PET or both ${ }^{11} \mathrm{C}$-PIB PET and ${ }^{18} \mathrm{~F}-\mathrm{AV} 45 \mathrm{PET}, 960$ were diagnosed with AD, 36 with frontotemporal dementia (FTD), 5 with dementia with Lewy bodies, 144 with $\mathrm{MCl}$, 29 with vascular dementia, 4 with corticobasal syndrome, and 15 with unclassifiable dementia. Baseline clinical diagnoses were independently established without access to PET imaging results. Apolipoprotein $\mathrm{E}(\mathrm{ApoE})$ genotype analysis was performed on $\mathrm{Cl}$ patients and 231 sex- and age-matched controls. Results: Of the $1,193 \mathrm{Cl}$ patients, $860(72.1 \%)$ were amyloid-positive. The prevalence of amyloid positivity in $\mathrm{AD}$ and $\mathrm{MCl}$ patients was $86.8 \%$ (833/ 960) and $9.7 \%$ (14/144), respectively. In FTD patients, the prevalence of $\beta$-amyloid deposits was $5.6 \%(2 / 36)$. In the 4 corticobasal syndrome patients, 2 were amyloid-positive. Three of the 5 patients with dementia with Lewy bodies showed amyloid positivity, as did 6 of the 29 vascular dementia (20.7\%) patients. The ApoEع4 allele frequency was significantly increased in amyloid-positive $\mathrm{Cl}$ patients $(30.5 \%)$ as compared with other amyloid-negative $\mathrm{Cl}$ patients (14\%) or controls (7.3\%). Conclusion: Amyloid imaging may potentially be the most helpful parameter for differential diagnosis in dementia, particularly to distinguish between AD and FTD. Amyloid PET can be used in conjunction with the ApoE\&4 allele genetic risk test for amyloid deposits.

Key Words: amyloid PET; dementia; multicenter

J Nucl Med 2020; 61:1814-1819

DOI: 10.2967/jnumed.119.240325

Received Dec. 2, 2019; revision accepted Mar. 28, 2020.

For correspondence or reprints contact: Yong Ji, Department of Neurology, Beijing Tiantan Hospital, Capital Medical University, No. 199, W. Section of S. Fourth Ring Rd., Fengtai District, Beijing, China 100070. E-mail: jiyongusa@126.com

${ }^{*}$ Contributed equally to this work.

Published online May 8, 2020.

COPYRIGHT (c) 2020 by the Society of Nuclear Medicine and Molecular Imaging.
B ecause of the aging of the world's population, the prevalence of dementia is increasing, with the number of individuals living with dementia currently estimated at 50 million worldwide and projected to increase to 75 million by 2030 . Moreover, the number of dementia cases is estimated to almost triple by 2050 (1). $\beta$-amyloid PET imaging allows in vivo detection of fibrillar plaques, a core neuropathologic feature of Alzheimer disease (AD). Several amyloid ligands have been studied in vivo, two of which, ${ }^{11} \mathrm{C}$-Pittsburgh compound $\mathrm{B}$ (PIB) (2) and ${ }^{18} \mathrm{~F}$-florbetapir (AV-45), are widely used in current clinical research (3) and have now been proven as reliable tools for assessing the amyloid burden in the brain of $\mathrm{AD}$ patients.

PET imaging with ${ }^{18} \mathrm{~F}\left({ }^{18} \mathrm{~F}-\mathrm{FDG}\right.$ PET) highlights the differential distribution of pathology in dementia disorders and has been used to study neurodegenerative diseases for over 3 decades $(4,5)$. $\mathrm{AD}$ causes hypometabolism predominantly in the posterior regions, including the posterior temporoparietal association cortex and posterior cingulate cortex $(6,7) .{ }^{18} \mathrm{~F}-\mathrm{FDG}$ PET images of patients with frontotemporal dementia (FTD) show decreased metabolism in the frontal and anterior temporal areas, cingulate gyri, uncus, insula, and subcortical areas, including the basal ganglia and medial thalamic regions (7-9).

Because of the invasive nature of lumbar puncture for the collection of cerebrospinal fluid, neuroimaging modalities such as ${ }^{18}$ F-FDG PET and ${ }^{11} \mathrm{C}-\mathrm{PIB}$ PET/AV-45 PET are more accepted in routine clinical practice to improve the diagnosis of dementia subtypes. To date, most amyloid PET studies have been conducted in single centers with smaller sample sizes. Therefore, we performed a multicenter study of patients from 5 clinics with 3 views in mind: to estimate the prevalence of amyloid positivity in a large sample encompassing a variety of dementia syndromes, to evaluate the association between ${ }^{18}$ F-FDG PET and amyloid scans in this cohort of patients, and to analyze the association between the apolipoprotein E (ApoE) $\varepsilon 4$ gene and amyloid deposits on PET scans.

\section{MATERIALS AND METHODS}

\section{Subjects}

In total, 1,193 consecutive patients with cognitive impairment (CI) were recruited at the PET/CT Center of Beijing Tiantan Hospital, the General Hospital of the People's Liberation Army, Shanghai Huashan 
Hospital, the Cognitive Clinic of Tianjin Huanhu Hospital, and the General Hospital of Tianjin Medical University between December 2012 and December 2018. All participants were 19-92 y old. The inclusion criteria were a clinical diagnosis of mild CI (MCI), dementia of any type or unclassifiable dementia, and imaging with ${ }^{11} \mathrm{C}$-PIB PET or ${ }^{18} \mathrm{~F}-\mathrm{AV} 45$ PET, with or without ${ }^{18} \mathrm{~F}$-FDG PET, within 1 mo of the initial clinical diagnosis. Sixty patients were excluded whose clinical data were not recorded in detail by their clinicians.

The clinical assessment was performed by neurologists experienced in dementia care and included a detailed history taken from the primary caregivers of the patient, a physical examination, a cognitive assessment, and laboratory studies, including a thyroid function test, vitamin B12 level, folate level, and syphilis serology.

Clinical criteria for AD, FTD, dementia with Lewy bodies (DLB), and vascular dementia $(\mathrm{VaD})$ were used to establish the initial clinical diagnosis without the use of any biomarker. The diagnosis of different dementia subtypes was based on the respective diagnostic guidelines using brain MRI and laboratory tests. Probable AD dementia was diagnosed according to the criteria of the National Institute on Aging and the Alzheimer Association workgroup (10). Patients with DLB were diagnosed using the criteria of McKeith et al. (11). Behavioral variant of FTD was diagnosed using revised diagnostic criteria reported by the International Behavioral Variant of FTD Criteria Consortium (12). Language variant of FTD and primary progressive aphasia were diagnosed according to the classification of primary progressive aphasia and its variants $(13,14)$. Corticobasal syndrome (CBS) was diagnosed according to the criteria published in 2013 (15). Patients with VaD were diagnosed according to the NINDS-AIREN criteria (National Institute of Neurologic Disorders and Stroke/Association International pour la Recherche et l'Enseignement en Neurosciences) (16). MCI was diagnosed according to the criteria published in 2004 (17). Subjects who had any contraindications to MRI or PET scanning were excluded from the study.

The ApoE genotype was determined from venous blood samples. Controls were group-matched to patients with respect to age and sex.

\section{Neuropsychologic Testing}

Subjects were examined using a battery of tests during the 2 wk before PET imaging. These tests included the Mini Mental State Examination (MMSE), Montreal Cognitive Assessment (MoCA), Activitiesof-Daily-Living Scale, and Clinical Dementia Rating.

\section{MRI Acquisition}

MRI scans were obtained on a 3.0-T GE Healthcare scanner or a 3.0-T Siemens Trio, A Tim, MRI scanner. The interval between MRI and amyloid PET was no longer than $2 \mathrm{wk}$. T1-weighted coronal images were acquired using a 3-dimensional spoiled gradient-recalled-echo inversion-recovery prepped sequence (1-mm slice thickness). All images were reconstructed to a matrix of $256 \times 256$, with an isotropic resolution of $1 \times 1 \times 1 \mathrm{~mm}$. These scans were used to define regions of interest for PET data analysis.

\section{PET Acquisition}

${ }^{11} \mathrm{C}$-PIB PET imaging was conducted at the PET/CT centers using a GE Healthcare Discovery LS PET/CT scanner or a Siemens Biograph mCT Flow PET/CT scanner (Huashan Hospital) in the 3-dimensional scanning mode. PIB was administered into an antecubital vein as a bolus injection at a mean dose of $370-555 \mathrm{MBq} .{ }^{11} \mathrm{C}-\mathrm{PIB}$ PET images were acquired during a 90-min dynamic PET scan. ${ }^{11} \mathrm{C}$-PIB uptake in each cortical region and across the whole cortex was calculated. The cerebellar cortex was chosen as the reference tissue. ${ }^{11} \mathrm{C}-\mathrm{PIB}$ integral images were coregistered to each subject's T1-weighted MR images. An MRI-based automated region-of-interest technique was used to sample each individual's PIB images. Imaging data at 40-60 min after injection were used for the analysis of PIB uptake to get parametric images of PIB SUV ratios. Patients were diagnosed as PIB-positive on the basis of both visual interpretations of elevated binding in the neocortex and semiquantitative assessment (SUV ratio > 1.40) (18).

${ }^{18} \mathrm{~F}-\mathrm{AV} 45$ PET scans were obtained on a Discovery Elite scanner (GE Healthcare) at Tiantan Hospital or a Siemens Biograph mCT Flow PET/ CT scanner at Huashan Hospital. ${ }^{18} \mathrm{~F}-\mathrm{AV} 45$ PET was performed for $20 \mathrm{~min}$, starting $50 \mathrm{~min}$ after injection of $248 \pm 58 \mathrm{MBq} .{ }^{18} \mathrm{~F}-\mathrm{AV} 45$ PET data were reconstructed using ordered-subset expectation maximization with weighted attenuation. Images were smoothed using a 5-mm gaussian kernel with scatter correction and were evaluated for patient motion and adequacy of statistical counts before analysis. SUV ratios were calculated using the cerebellar gray matter reference region to normalize mean activity from 50 to $70 \mathrm{~min}$. Patients were diagnosed as AV45-positive on the basis of both visual interpretations of elevated binding in the neocortex and semiquantitative assessment (SUV ratio $>$ 1.11).

Subjects were injected intravenously with $240-333 \mathrm{MBq}$ of ${ }^{18} \mathrm{~F}-$ FDG, and a 10-min static PET scan was obtained 40 min after injection of ${ }^{18} \mathrm{~F}$-FDG. Voxel-based statistical analysis was performed on ${ }^{18} \mathrm{~F}-\mathrm{FDG}$ PET images using Statistical Parametric Mapping, version 8, and MATLAB, version 2010b (MathWorks), for Microsoft Windows. Regions that reached an uncorrected $P$ value of less than 0.001 were considered statistically significant. Anatomic localization was based on the superimposition of Statistical Parametric Mapping T maps onto the ch2bet template brain and identification of the localization using AAL (automated anatomic labeling) software and anatomic atlases (http://www. talairach.org/) (19). The findings were rendered using the publicly available MRIcron software (https://www.nitrc.org/projects/mricron).

\section{Statistical Analysis}

Categoric variables were examined using the $\chi^{2}$ or Fisher exact test. Data are expressed as the median, mean $\pm \mathrm{SD}$, or a number and percentage. ANOVA and independent Student $t$ tests were used to determine statistical differences between images and in the duration of symptoms between amyloid-positive and -negative patients. $P$ values of less than 0.05 were considered statistically significant.

\section{Ethics}

Detailed written informed consent was obtained from all subjects or their relatives. The present study was approved by the Ethics Committees of Tianjin Huanhu Hospital, Beijing Tiantan Hospital, the General Hospital of the People's Liberation Army, Shanghai Huashan Hospital, and Tianjin Medical University. The procedures were performed in accordance with the ethical standards of the Committee on Human Experimentation.

\section{RESULTS}

In total, 1,193 patients ( $55.4 \%$ male) were recruited, of whom 960 had AD, 36 had FTD, 5 had DLB, 4 had CBS, 29 had VaD, 144 had MCI, and 15 had unclassifiable dementia. ${ }^{11} \mathrm{C}$-PIB PET data were available for $562(47.1 \%)$ patients, ${ }^{18} \mathrm{~F}$-AV45 PET data were available for $643(53.9 \%)$ patients, and both ${ }^{18}$ F-FDG PET and ${ }^{11} \mathrm{C}$-PIB PET or ${ }^{18} \mathrm{~F}$-AV45 PET data were available for 616 $(51.6 \%)$ patients. Of the 12 patients with both ${ }^{11} \mathrm{C}$-PIB PET and ${ }^{18} \mathrm{~F}-\mathrm{AV} 45$ PET scans, 3 were both PIB- and AV45-negative, and 9 were positive for both. The observations on the ${ }^{11} \mathrm{C}$-PIB and ${ }^{18} \mathrm{~F}$ AV45 PET scans were consistent in these 12 patients.

Table 1 displays the demographic characteristics of all patients with CI. Most patients (85.2\%) were 50-79 y old.

Table 2 shows the probability of amyloid positivity on PET imaging across diagnostic and age groups. Of the 1,193 CI patients, 860 $(72.1 \%)$ were amyloid-positive. The prevalence of amyloid positivity in AD and MCI was 86.8\% (833/960) and 9.7\% (14/144), 


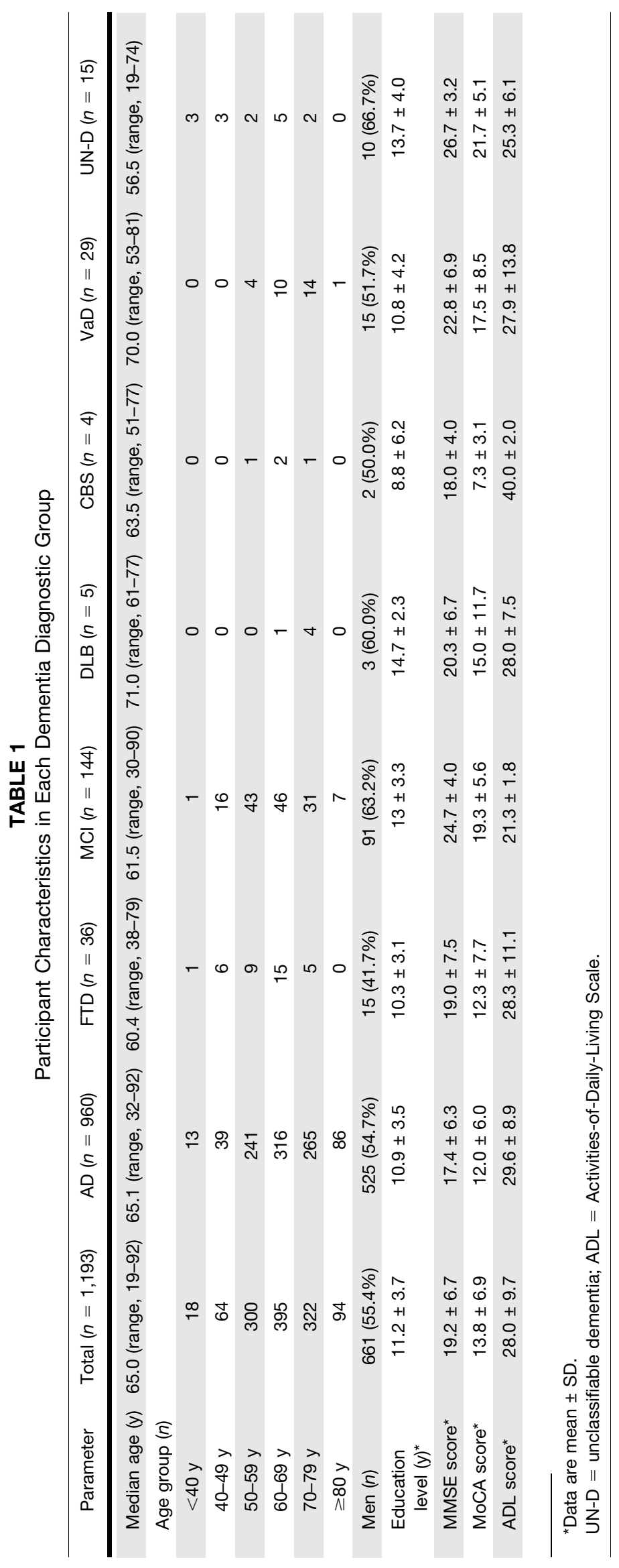




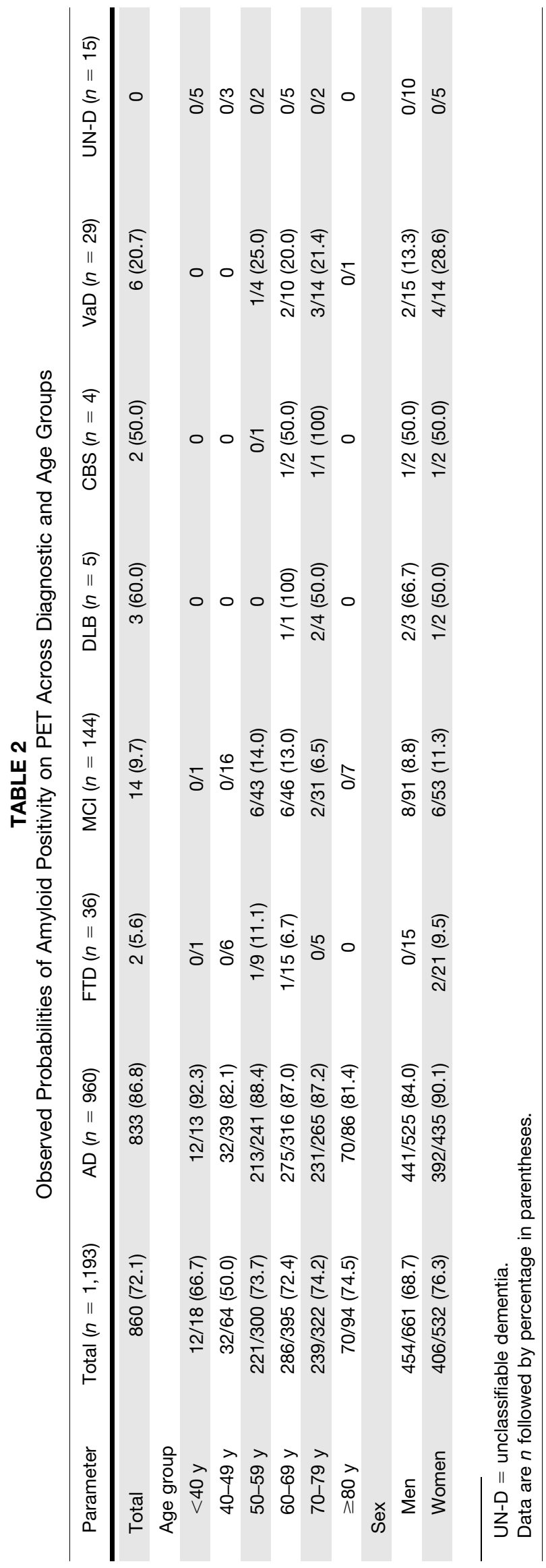

respectively. In FTD patients, the prevalence of $\beta$-amyloid deposits was $5.6 \%$ (2/36). Of the 4 CBS patients, 2 were amyloid-positive, as were 3 of the 5 DLB patients and 6 of the $29(20.7 \%) \mathrm{VaD}$ patients. All 15 patients with unclassifiable CI were amyloid-negative. In AD patients, those who were amyloid-positive had lower MMSE and MoCA scores than those who were amyloid-negative (MMSE: $16.8 \pm 6.1$ vs. $21.3 \pm 5.7, P=0.001$; MoCA: $11.6 \pm 5.8$ vs. $14.9 \pm 6.8, P=0.024)$. In CBS, DLB, and VaD patients, those who were amyloid-positive also had lower MMSE and MoCA scores than those who were amyloid-negative (MMSE: $19.2 \pm 6.3$ vs. $23.9 \pm$ $6.1, P=0.036$; MoCA: $13.7 \pm 6.1$ vs. $16.9 \pm 7.0, P=0.032$ ).

Table 3 shows that the prevalence of amyloid positivity in all CI patients was significantly different among age groups, but in $\mathrm{AD}$ patients, this difference did not exist.

Of all the CI patients, ${ }^{18} \mathrm{~F}-\mathrm{FDG}$ PET data were available for $463 \mathrm{AD}$ and 36 FTD patients. Of all the clinically diagnosed AD patients, 80.6\% (373/463) showed AD-pattern hypometabolism predominantly in the posterior regions, including the posterior temporoparietal association cortex and posterior cingulate cortex, with or without frontal lobe involvement. In all 396 clinical AD patients with amyloid-positive findings, $340(85.9 \%)$ showed AD-pattern hypometabolism, and in all 373 clinical AD patients with AD-pattern hypometabolism, 340 (91.2\%) showed amyloid positivity. A total of $4.5 \%$ (21/463) showed hypometabolism in the frontal and anterior temporal areas, cingulate gyri, and insula, and of these patients only 12 $(57.1 \%)$ were amyloid-positive. A total of $14.9 \%(69 / 463)$ showed nonspecific hypometabolism, but only 44 (63.7\%) were amyloid-positive (Supplemental Table 1; supplemental materials are available at http://jnm.snmjournals.org).

Of the 36 FTD patients, $77.8 \%$ (28/36) showed FTD-pattern hypometabolism predominantly in the anterior regions, including the frontal and anterior temporal areas, anterior cingulate gyrus, and insula; $13.9 \%$ (5/36) showed AD-pattern hypometabolism; and $8.3 \%$ (3/36) showed nonspecific hypometabolism. Only 2 (40\%) FTD patients with AD-pattern hypometabolism were amyloidpositive (Supplemental Table 2).

The frequency of the ApoE\&4 allele was $30.5 \%$ in $\beta$-amyloidpositive $\mathrm{CI}, 14.0 \%$ in $\beta$-amyloid-negative $\mathrm{CI}$, and $7.3 \%$ in the control. The ApoEs4 allele frequency was significantly higher in CI patients with amyloid positivity than in CI patients with amyloid negativity (Supplemental Table 3).

\section{DISCUSSION}

The main findings of the present multicenter PET imaging study are that the prevalence of amyloid deposition on PET images was $86.8 \%$ in clinically diagnosed AD patients, $9.7 \%$ in MCI patients, and $5.6 \%$ in FTD patients. Of the 4 CBS patients, 2 were amyloidpositive, as were 3 of the $5(60 \%)$ DLB patients and 6 of the 29 $(20.7 \%) \mathrm{VaD}$ patients. The significant difference in amyloid positivity across dementia types suggests that amyloid imaging may potentially be the most helpful parameter for differential diagnosis in dementia, particularly in distinguishing between AD and FTD. Furthermore, amyloid deposits in non-AD dementia, including CBS, DLB, and VaD, may be clinically important, since amyloid positivity was associated with worse global cognition.

In the present study, amyloid negativity on PET was observed in $13.2 \%$ of clinically diagnosed $\mathrm{AD}$ patients, a percentage that is consistent with a system metaanalysis performed in 2015 (20). Of our clinically diagnosed AD patients aged 80 y or older, $81.4 \%$ were amyloid-positive, a percentage that is lower than the $88.4 \%$ seen in 
TABLE 3

Prevalence of Amyloid Positivity on PET Scans Across Diagnostic, Sex, and Age Groups

\begin{tabular}{|c|c|c|}
\hline Parameter & Amyloid-positive & $P$ \\
\hline Diagnosis & & $<0.001$ \\
\hline$A D$ & $833(86.7)$ & \\
\hline FTD & $2(5.6)$ & \\
\hline $\mathrm{MCl}$ & $14(9.7)$ & \\
\hline $\mathrm{DLB}+\mathrm{CBS}$ & $5(55.6)$ & \\
\hline $\mathrm{VaD}$ & $6(20.7)$ & \\
\hline \multicolumn{3}{|l|}{ Total group } \\
\hline Age group & & 0.004 \\
\hline$<40 y$ & $12(66.7)$ & \\
\hline $40-49$ y & $32(50.0)$ & \\
\hline $50-59 y$ & $221(73.7)$ & \\
\hline $60-69$ y & $286(72.4)$ & \\
\hline $70-79$ y & 239 (74.2) & \\
\hline$\geq 80 y$ & $70(74.5)$ & \\
\hline Sex & & 0.002 \\
\hline Men & $454(68.7)$ & \\
\hline Women & 406 (76.3) & \\
\hline \multicolumn{3}{|l|}{$A D$ group } \\
\hline Age group & & 0.569 \\
\hline$<40 \mathrm{y}$ & 12 (92.3) & \\
\hline $40-49$ y & $32(82.1)$ & \\
\hline $50-59$ y & $213(88.4)$ & \\
\hline $60-69 y$ & $275(87.0)$ & \\
\hline $70-79$ y & 231 (87.2) & \\
\hline$\geq 80 y$ & $70(81.4)$ & \\
\hline Sex & & 0.004 \\
\hline Men & $441(83.4)$ & \\
\hline Women & $392(90.1)$ & \\
\hline
\end{tabular}

Data are $n$ followed by percentage in parentheses.

the 50- to 59-y-old patients. The AD phenocopy was most prevalent in older patients and may best be explained by a mixture of agerelated conditions (e.g., hippocampal sclerosis, argyrophilic grain disease, or tangle-predominant dementia (21-23)) that preferentially target the limbic system, resulting in a memory-predominant presentation that may be mistaken for $\mathrm{AD}$, in addition to falsenegative PET findings. This problem requires more tracers to further detect the pathologic changes in vivo.

Of the 4 clinically diagnosed CBS patients, 2 showed amyloid positivity, which is consistent with a change to AD pathology. In recent years, CBS with $\mathrm{AD}$ pathology has been reported in pathology and neuroimaging studies (24-27). Because of this clinicopathologic diversity, Boeve et al. introduced the term $C B S$ to distinguish the clinical syndrome from the pathologic entity, corticobasal degeneration (28). Amyloid PET imaging is the optimal modality for the detection of AD pathology in CBS patients and can direct future medical treatment.

Cerebrovascular disease is the second most common cause of age-related $\mathrm{CI}$ and dementia and is widely recognized as $\mathrm{VaD}$
(29). Autopsy findings have revealed AD-type pathologic changes $(30,31)$ in VaD patients. For instance, a U.S. study (32) reported that $87 \%$ of patients enrolled to examine $\mathrm{VaD}$ in a dementia clinic setting were found to have $\mathrm{AD}$, either alone $(58 \%)$ or in combination with cerebrovascular disease $(42 \%)$. Of our $29 \mathrm{VaD}$ patients, $6(20.7 \%)$ were amyloid-positive, indicating that these patients have $\mathrm{AD}$ alone or in combination with $\mathrm{VaD}$.

Of our 144 MCI patients, only $9.7 \%$ showed amyloid positivity. The rate of amyloid positivity was lower than in previous studies, which have reported that $41 \%-75 \%$ of $\mathrm{MCI}$ patients show $\beta$-amyloid retention on amyloid PET imaging (33-35). The criteria provided for MCI by Winblad et al. (17) and used in our study are relatively general, as may explain the low rate of amyloid positivity we found in MCI patients.

Of all the clinically diagnosed $\mathrm{AD}$ patients in the present study, $80.6 \%$ showed hypometabolism predominantly in the posterior regions, including the posterior temporoparietal association cortex and posterior cingulate cortex, with or without frontal lobe involvement. Retrospective studies have illustrated that ${ }^{18} \mathrm{~F}$-FDG PET has 94\% sensitivity and $73 \%$ specificity for predicting AD pathology (36). Hypometabolic regions spread to the frontal association cortices in moderate to severe $\mathrm{AD}$.

Of the 36 FTD patients, $77.8 \%$ showed FTD-pattern hypometabolism, $13.9 \%$ showed AD-pattern hypometabolism, and $8.3 \%$ showed nonspecific hypometabolism. In FTD patients, frontal and temporal regions and the striatum and thalamus show decreased glucose metabolism (37). Metabolic and morphologic changes occur bilaterally in the frontal and temporal lobes, whereas regions of metabolism are more severely affected than regions of atrophy in the frontal lobe (38). As the disease progresses to advanced stages, hypometabolism spreads from localized frontal lobe areas to the parietal and temporal cortices in some patients (39); thus, the advanced stage may mimic frontal-variant AD-pattern hypometabolism. Therefore, ${ }^{18}$ F-FDG PET could be used to differentiate between a diagnosis of FTD and AD in patients with mild to moderate dementia.

ApoE\&4 as a strong risk factor for $\mathrm{AD}$ has been studied for many years. In our 150 patients, the frequency of the ApoEc4 allele was $30.5 \%$ in those with amyloid-positive CI, a percentage that was higher than that in patients with amyloid-negative CI and the controls. Human and animal studies have shown that brain $\beta$-amyloid levels and plaque loads are ApoE isoformdependent $(\varepsilon 4>\varepsilon 3>\varepsilon 2)$, suggesting that ApoE isoforms differentially affect $\beta$-amyloid aggregation, clearance, and deposition (40-42). The metaanalysis showed that the likelihood of amyloid positivity was associated with age and ApoE\&4 status. In most non-AD dementia types, amyloid positivity increases with both age $(60-80$ y) and ApoEe4 carriership (20). A recent study showed that the ApoEc4 genotype influences the brain-amyloid deposition pattern, with the ApoE genotype and age being associated with an increased $\beta$-amyloid deposition rate (43). The main limitation of the present study is that only 160 patients were tested for ApoE genotype; thus, we could not analyze the relationship among age, ApoE genotype, and amyloid deposition pattern in detail.

\section{CONCLUSION}

Among patients with dementia, the significant difference in amyloid positivity across dementia types suggests that amyloid imaging may potentially be the most helpful parameter for differential diagnosis in dementia, particularly in distinguishing between AD and FTD. 
Furthermore, amyloid deposits in non-AD dementia, including CBS, $\mathrm{DLB}$, and $\mathrm{VaD}$, may be clinically important, since amyloid positivity was associated with worse global cognition. The ApoEc4 allele is a genetic risk factor for amyloid deposits.

\section{DISCLOSURE}

The present study was supported by the Natural Science Foundation of China (funding number 81571057) and the Tianjin Science and Technology Project (funding number 16ZXMJSY00010). No other potential conflict of interest relevant to this article was reported.

\section{KEY POINTS}

QUESTION: Is amyloid PET imaging a valuable test for the differential diagnosis of a variety of dementia syndromes?

PERTINENT FINDINGS: In our multicenter study including 1,193 patients with $\mathrm{Cl}, 860$ were amyloid-positive. Among patients with dementia, the prevalence of amyloid positivity was significantly different across dementia types.

IMPLICATIONS FOR PATIENT CARE: Amyloid imaging may potentially be the most helpful parameter for differential diagnosis in dementia, particularly in distinguishing between AD and FTD.

\section{REFERENCES}

1. 10 facts on dementia. World Health Organization website. https://www.who.int/ features/factfiles/dementia/en/. Updated September, 2019. Accessed July 7, 2020.

2. Klunk WE, Engler H, Nordberg A, et al. Imaging brain amyloid in Alzheimer's disease with Pittsburgh compound-B. Ann Neurol. 2004;55:306319.

3. Clark CM, Schneider JA, Bedell BJ, et al. Use of florbetapir-PET for imaging beta-amyloid pathology. JAMA. 2011;305:275-283.

4. Benson DF, Kuhl DE, Phelps ME, Cummings JL, Tsai SY. Positron emission computed tomography in the diagnosis of dementia. Trans Am Neurol Assoc. 1981;106: 68-71.

5. Alavi A, Reivich M, Ferris S, et al. Regional cerebral glucose metabolism in aging and senile dementia as determined by F-deoxyglucose and positron emission tomography. Exp Brain Res. 1982;(suppl 5):187-195.

6. Bohnen NI, Djang DSW, Herholz K, Anzai Y, Minoshima S. Effectiveness and safety of ${ }^{18}$ F-FDG PET in the evaluation of dementia: a review of the recent literature. J Nucl Med. 2012;53:59-71.

7. Herholz K, Boecker H, Nemeth I, Dunn G. FDG PET in dementia multicenter studies and clinical trials. Clin Transl Imaging. 2013;1:261-270.

8. Kamo H, McGeer PL, Harrop R, et al. Positron emission tomography and histopathology in Pick's disease. Neurology. 1987;37:439-445.

9. Ishii K, Sakamoto S, Sakaki M, et al. Cerebral glucose metabolism in patients with frontotemporal dementia. J Nucl Med. 1998;39:1875-1878.

10. McKhann GM, Knopman DS, Chertkow H, et al. The diagnosis of dementia due to Alzheimer's disease: recommendations from the National Institute on AgingAlzheimer's Association workgroups on diagnostic guidelines for Alzheimer's disease. Alzheimers Dement. 2011;7:263-269.

11. McKeith IG, Dickson DW, Lowe J, et al. Diagnosis and management of dementia with Lewy bodies: third report of the DLB Consortium. Neurology. 2005;65: 1863-1872.

12. Rascovsky K, Hodges JR, Knopman D, et al. Sensitivity of revised diagnostic criteria for the behavioural variant of frontotemporal dementia. Brain. 2011;134: 2456-2477.

13. Harris JM, Gall C, Thompson JC, et al. Classification and pathology of primary progressive aphasia. Neurology. 2013;81:1832-1839.

14. Gorno-Tempini ML, Hillis AE, Weintraub S, et al. Classification of primary progressive aphasia and its variants. Neurology. 2011;76:1006-1014.

15. Armstrong MJ, Litvan I, Lang AE, et al. Criteria for the diagnosis of corticobasal degeneration. Neurology. 2013;80:496-503.

16. Román GC, Tatemichi TK, Erkinjuntti T, et al. Vascular dementia: diagnostic criteria for research studies. Report of the NINDS-AIREN International Workshop. Neurology. 1993;43:250-260.
17. Winblad B, Palmer K, Kivipelto M, et al. Mild cognitive impairment: beyond controversies, towards a consensus-report of the International Working Group on Mild Cognitive Impairment. J Intern Med. 2004;256: 240-246.

18. Villeneuve S, Rabinovici GD, Cohn-Sheehy BI, et al. Existing Pittsburgh compound-B positron emission tomography thresholds are too high: statistical and pathological evaluation. Brain. 2015;138:2020-2033.

19. Morbelli S, Perneczky R, Drzezga A, et al. Metabolic networks underlying cognitive reserve in prodromal Alzheimer disease: a European Alzheimer Disease Consortium project. J Nucl Med. 2013;54:894-902.

20. Ossenkoppele R, Jansen WJ, Rabinovici GD, et al. Prevalence of amyloid PET positivity in dementia syndromes: a meta-analysis. JAMA. 2015;313:1939-1949.

21. Barkhof F, Polvikoski TM, van Straaten EC, et al. The significance of medial temporal lobe atrophy: a postmortem MRI study in the very old. Neurology. 2007; 69:1521-1527.

22. Serrano-Pozo A, Qian J, Monsell SE, et al. Mild to moderate Alzheimer dementia with insufficient neuropathological changes. Ann Neurol. 2014;75:597601

23. Crary JF, Trojanowski JQ, Schneider JA, et al. Primary age-related tauopathy (PART): a common pathology associated with human aging. Acta Neuropathol (Berl). 2014;128:755-766.

24. Armstrong RA. Size frequency distributions of $\beta$-amyloid $(A \beta)$ deposits: a comparative study of four neurodegenerative disorders. Folia Neuropathol. 2012;50: 240-249.

25. Armstrong RA. A comparison of the spatial patterns of $\beta$-amyloid (A $\beta)$ deposits in five neurodegenerative disorders. Folia Neuropathol. 2018;56:284-292.

26. Wilson H, Pagano G, Politis M. Dementia spectrum disorders: lessons learnt from decades with PET research. J Neural Transm. 2019;126:233-251.

27. Parmera JB, Rodriguez RD, Studart Neto A, Nitrini R, Brucki SMD. Corticobasal syndrome: a diagnostic conundrum. Dement Neuropsychol. 2016;10:267275.

28. Boeve BF, Lang AE, Litvan I. Corticobasal degeneration and its relationship to progressive supranuclear palsy and frontotemporal dementia. Ann Neurol. 2003; 54(suppl):S15-S19.

29. Helman AM, Murphy MP. Vascular cognitive impairment: modeling a critical neurologic disease in vitro and in vivo. Biochim Biophys Acta. 2016;1862:975982.

30. Erkinjuntti T, Haltia M, Palo J, Sulkava R, Paetau A. Accuracy of the clinical diagnosis of vascular dementia: a prospective clinical and post-mortem neuropathological study. J Neurol Neurosurg Psychiatry. 1988;51:1037-1044.

31. Hulette C, Nochlin D, McKeel D, et al. Clinical-neuropathologic findings in multi-infarct dementia: a report of six autopsied cases. Neurology. 1997;48: 668-672.

32. Nolan KA, Lino MM, Seligmann AW, Blass JP. Absence of vascular dementia in an autopsy series from a dementia clinic. J Am Geriatr Soc. 1998;46:597-604.

33. Wolk DA, Price JC, Saxton JA, et al. Amyloid imaging in mild cognitive impairment subtypes. Ann Neurol. 2009;65:557-568.

34. Kim JY, Lim JH, Jeong YJ, Kang DY, Park KW. The effect of clinical characteristics and subtypes on amyloid positivity in patients with amnestic mild cognitive impairment. Dement Neurocogn Disord. 2019;18:130-137.

35. Tomadesso C, de La Sayette V, de Flores R, et al. Neuropsychology and neuroimaging profiles of amyloid-positive versus amyloid-negative amnestic mild cognitive impairment patients. Alzheimers Dement (Amst). 2018;10:269-277.

36. Silverman DH, Small GW, Chang CY, et al. Positron emission tomography in evaluation of dementia: regional brain metabolism and long-term outcome. JAMA. 2001;286:2120-2127.

37. Ishii K, Sakamoto S, Sasaki M, et al. Cerebral glucose metabolism in patients with frontotemporal dementia. J Nucl Med. 1998;39:1875-1878.

38. Engler H, Santillo AF, Wang SX, et al. In vivo amyloid imaging with PET in frontotemporal dementia. Eur J Nucl Med Mol Imaging. 2008;35:100-106.

39. Poljansky S, Ibach B, Hirschberger B, et al. A visual $\left[{ }^{18} \mathrm{~F}\right]$ FDG-PET rating scale for the differential diagnosis of frontotemporal lobar degeneration. Eur Arch Psychiatry Clin Neurosci. 2011;261:433-446.

40. Reiman EM, Chen K, Liu X, et al. Fibrillar amyloid-beta burden in cognitively normal people at 3 levels of genetic risk for Alzheimer's disease. Proc Natl Acad Sci USA. 2009;106:6820-6825.

41. Castellano JM, Kim J, Stewart FR, et al. Human apoE isoforms differentially regulate brain amyloid- $\beta$ peptide clearance. Sci Transl Med. 2011;3:89ra57.

42. Bales KR, Liu F, Wu S, et al. Human APOE isoform-dependent effects on brain beta-amyloid levels in PD APP transgenic mice. J Neurosci. 2009;29:6771-6779.

43. Toledo JB, Habes M, Sotiras A, et al. APOE effect on amyloid- $\beta$ PET spatial distribution, deposition rate, and cut-points. J Alzheimers Dis. 2019;69:783-793. 